\title{
In silico modeling predicts drug sensitivity of patient-derived cancer cells
}

\author{
Sandeep C Pingle ${ }^{1 \dagger}$, Zeba Sultana ${ }^{2 \dagger}$, Sandra Pastorino ${ }^{1}$, Pengfei Jiang ${ }^{1}$, Rajesh Mukthavaram ${ }^{1}$, Ying Chao ${ }^{1}$, \\ Ila Sri Bharati ${ }^{1}$, Natsuko Nomura', Milan Makale', Taher Abbasi ${ }^{3}$, Shweta Kapoor ${ }^{2}$, Ansu Kumar ${ }^{2}$, \\ Shahabuddin Usmani ${ }^{2}$, Ashish Agrawal ${ }^{2}$, Shireen Vali ${ }^{2,3}$ and Santosh Kesari ${ }^{1,4^{*}}$
}

\begin{abstract}
Background: Glioblastoma (GBM) is an aggressive disease associated with poor survival. It is essential to account for the complexity of GBM biology to improve diagnostic and therapeutic strategies. This complexity is best represented by the increasing amounts of profiling ("omics") data available due to advances in biotechnology. The challenge of integrating these vast genomic and proteomic data can be addressed by a comprehensive systems modeling approach.

Methods: Here, we present an in silico model, where we simulate GBM tumor cells using genomic profiling data. We use this in silico tumor model to predict responses of cancer cells to targeted drugs. Initially, we probed the results from a recent hypothesis-independent, empirical study by Garnett and co-workers that analyzed the sensitivity of hundreds of profiled cancer cell lines to 130 different anticancer agents. We then used the tumor model to predict sensitivity of patient-derived GBM cell lines to different targeted therapeutic agents.

Results: Among the drug-mutation associations reported in the Garnett study, our in silico model accurately predicted $\sim 85 \%$ of the associations. While testing the model in a prospective manner using simulations of patient-derived GBM cell lines, we compared our simulation predictions with experimental data using the same cells in vitro. This analysis yielded a 75\% agreement of in silico drug sensitivity with in vitro experimental findings.

Conclusions: These results demonstrate a strong predictability of our simulation approach using the in silico tumor model presented here. Our ultimate goal is to use this model to stratify patients for clinical trials. By accurately predicting responses of cancer cells to targeted agents a priori, this in silico tumor model provides an innovative approach to personalizing therapy and promises to improve clinical management of cancer.
\end{abstract}

Keywords: Glioblastoma, Cancer, In Silico modeling, Deterministic model, Virtual tumor technology, Tumor profiling, Personalized therapy, Targeted therapy

\section{Introduction}

Cancer remains a major unmet clinical need despite advances in clinical medicine and cancer biology. Glioblastoma $(\mathrm{GBM})$ is the most common type of primary adult brain cancer, characterized by infiltrative cellular proliferation, angiogenesis, resistance to apoptosis, and widespread genomic aberrations. GBM patients have poor prognosis, with a median survival of 15 months [1]. Molecular profiling and

\footnotetext{
* Correspondence: skesari@ucsd.edu

${ }^{\dagger}$ Equal contributors

'Translational Neuro-Oncology Laboratories, Moores Cancer Center, UC San Diego, La Jolla, CA 92093, USA

${ }^{4}$ Department of Neurosciences, UC San Diego, La Jolla, CA 92093, USA Full list of author information is available at the end of the article
}

genome-wide analyses have revealed the remarkable genomic heterogeneity of GBM [2,3]. Based on tumor profiles, GBM has been classified into four distinct molecular subtypes [4]. However, even with existing molecular classifications, the high intertumoral heterogeneity of GBM makes it difficult to predict drug responses a priori. This is even more evident when trying to predict cellular responses to multiple signals following combination therapy. Our rationale is that a systems-driven computational approach will help decipher pathways and networks involved in treatment responsiveness and resistance.

Though computational models are frequently used in biology to examine cellular phenomena, they are not 
common in cancers, particularly brain cancers $[5,6]$. However, models have previously been used to estimate tumor infiltration following surgery [7] or changes in tumor density following chemotherapy in brain cancers [8]. More recently, brain tumor models have been used to determine the effects of conventional therapies including chemotherapy and radiation [5]. Brain tumors have also been studied using an agent-based modeling approach [9]. Multiscale models that integrate hierarchies in different scales are being developed for application in clinical settings [10]. Unfortunately, none of these models have been successfully translated into the clinic so far. It is clear that innovative models are required to translate data involving biological networks and genomics/proteomics into optimal therapeutic regimens. To this end, we present a deterministic in silico tumor model that can accurately predict sensitivity of patient-derived tumor cells to various targeted agents.

\section{Methods}

Description of In Silico model (Version 7.3 Cellworks)

We performed simulation experiments and analyses using the predictive tumor model - a comprehensive and dynamic representation of signaling and metabolic pathways in the context of cancer physiology. This in silico model includes representation of important signaling pathways implicated in cancer such as growth factors such as EGFR, PDGFR, FGFR, c-MET, VEGFR and IGF-1R; cytokine and chemokines such as IL1, IL4, IL6, IL12, TNF; GPCR mediated signaling pathways; mTOR signaling; cell cycle regulations, tumor metabolism, oxidative and ER stress, representation of autophagy and proteosomal degradation, DNA damage repair, p53 signaling and apoptotic cascade. The current version of this model includes more than 4,700 intracellular biological entities and $\sim 6,500$ reactions representing their interactions, regulated by $\sim 25,000$ kinetic parameters. This comprises a comprehensive and extensive coverage of the kinome, transcriptome, proteome and metabolome. Currently, we have 142 kinases and 102 transcription factors modeled in the system.

\section{Model development}

We built the basic model by manually curating data from the literature and aggregating functional relationships between proteins. The detailed procedure for model development is explained in Additional file 1 (Section 2) using the example of the epidermal growth factor receptor (EGFR) pathway block (Additional file 1: Figure S1 and Figure S2). We have also presented examples of how the kinetic parameters are derived from experimental data, in Additional file 1: (Section 2). We have validated the simulation model prospectively and retrospectively, at phenotype and biomarker levels using extensive in vitro and in vivo studies [11-20].

\section{Disease phenotype definitions}

Disease phenotype indices are defined in the tumor model as functions of biomarkers involved. Proliferation Index is an average function of the active CDK-Cyclin complexes that define cell cycle check-points and are critical for regulating overall tumor proliferation potential. The biomarkers included in calculating this index are: CDK4-CCND1, CDK2-CCNE, CDK2-CCNA and CDK1-CCNB1. These biomarkers are weighted and their permutations provide an index definition that gives maximum correlation with experimentally reported trend for cellular proliferation (based on literature).

We also generate a Viability Index based on 2 subindices: Survival Index and Apoptosis Index. The biomarkers constituting the Survival Index include: AKT1, BCL2, MCL1, BIRC5, BIRC2 and XIAP. These biomarkers support tumor survival. The Apoptosis Index comprises: BAX, CASP3, NOXA and CASP8. The overall Viability Index of a cell is calculated as a ratio of Survival Index/ Apoptosis Index. The weightage of each biomarker is adjusted so as to achieve a maximum correlation with the experimental trends for the endpoints (based on literature).

In order to correlate the results from experiments such as MTT Assay, which are a measure of metabolically active cells, we have a 'Relative Growth' Index that is an average of the Survival and Proliferation Indices.

The percent change seen in these indices following a therapeutic intervention helps assess the impact of that particular therapy on the tumor cell. A cell line in which the Proliferation/Viability Index decreases by $<20 \%$ from the baseline is considered resistant to that particular therapy.

\section{Creation of cancer cell line and its variants}

To create a cancer-specific simulation model, we start with a representative non-transformed epithelial cell as control. This cell is triggered to transition into a neoplastic state, with genetic perturbations like mutation and copy number variation (CNV) known for that specific cancer model. We also created in silico variants for cancer cell lines, to test the effect of various mutations on drug responsiveness. We created these variants by adding or removing specific mutations from the cell line definition. For example, DU145 prostate cancer cells normally have RB1 deletion. To generate a variant of DU145 with wild-type RB1 (WT), we retained the rest of its mutation definition except for the RB1 deletion, which was converted to WT RB1 (Additional file 1).

\section{Simulation of drug effect}

To simulate the effect of a drug in the in silico tumor model, the targets and mechanisms of action of the drug are determined from published literature. The drug concentration is 
assumed to be post-ADME (Absorption, Distribution, Metabolism and Excretion).

\section{Creation of simulation avatars of patient-derived GBM cell lines}

To predict drug sensitivity in patient-derived GBM cell lines, we created simulation avatars (in silico profiles) for each cell line as illustrated in Figure 1B. First, we simulated the network dynamics of GBM cells by using experimentally determined expression data (Additional file 1: Table S1; Additional file 1: Section 7). Next, we overlay tumor-specific genetic perturbations on the control network, in order to dynamically generate the simulation avatar. For instance, the patient-derived cell line SK987 is characterized by overexpression of AKT1, EGFR, IL6, and PI3K among other proteins and knockdown of CDKN2A, CDKN2B, RUNX3, etc. (Additional file 1: Table S1). After adding this information to the model, we further optimized the magnitude of the genetic perturbations, based on the responses of this simulation avatar to three molecularly targeted agents: erlotinib, sorafenib and dasatinib. The response of the cells to these drugs (from in vitro experimental data) was used as an "alignment data set". In this manner, we used "alignment drugs" (erlotinib, sorafenib, and dasatinib) to optimize the magnitude of genetic perturbation in the trigger files and their impact on key pathways targeted by these drugs. For example, most GBM cell lines demonstrated dominance of EGFR signaling as they had gains in copy number of EGFR gene. Hence the effect of EGFR inhibitor would be a good indicator for the relative dominance of this signaling pathway. This is illustrated in further details in Additional file 1 using an example of two cell line profiles that have EGFR over-expression but differential response to EGFR inhibitor. Similarly, sorafenib helped determine and align with MEK/ERK activation, while dasatinib with activation of SRC signaling.

\section{Simulation protocol}

The simulation protocol included 3 states:

1. Control State - The in silico model was simulated for 50,000 seconds, during which the different biological entities (called species) attain a steady-state concentration. This concentration depends on the balance between the rate of reaction nodes producing the species and the reaction nodes utilizing/degrading the species. This is an untriggered system and is representative of a non-transformed epithelial cell.

2. Disease State - At 50,000 seconds simulation time, we introduced the mutation data (specific to patient-derived GBM cell lines to be created) and simulated for an additional 1,25,000 seconds. During this time, the system attained a new steady state that aligns to the network dynamics of the cell line.

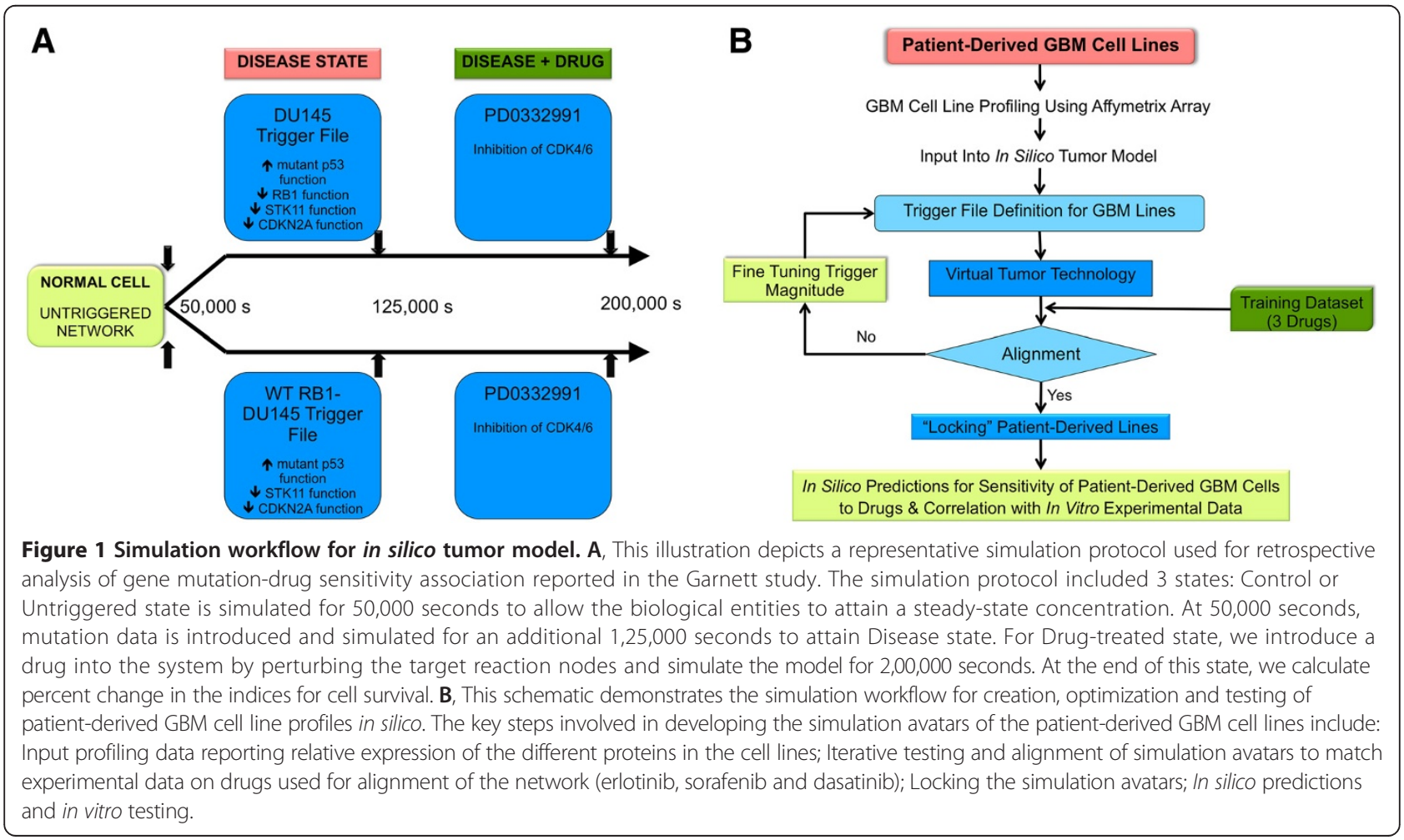


3. Disease State + Drug Treatment - Following the simulation run time of 1,25,000 seconds for Disease State, we introduced the drug into the system by perturbing the target reaction nodes as explained above. We then simulated the model further for 2,00,000 seconds (drug treatment). A percent change in the indices for cell survival (described earlier) indicates the therapeutic potential of the drug. Iterative simulations with varying concentrations of the drug generate dose-response curves from which $\mathrm{IC}_{50}$ values can be determined.

Figure $1 \mathrm{~A}$ is a schematic of the representative simulation protocol that we used for the retrospective analysis of gene mutations-drug effects reported in the study by Garnett and co-workers. Figure 1B illustrates the workflow for simulation studies on patient-derived GBM cell lines. For the patient-derived GBM cell line predictions, we prospectively compared in silico responses to experimentally obtained results (in vitro data from patientderived GBM cell lines) and determined corroboration between in silico and in vitro data. As per the doseresponse plots generated by in silico predictions, a cell line was considered sensitive to a drug if it demonstrated $>20 \%$ decrease in relative growth. The 20\% threshold was used for both in silico predictions and for in vitro experimental data.

\section{Patient-derived glioblastoma cell lines}

Fresh human glioblastoma samples were acquired from brain tumor patients undergoing clinically indicated surgery (University of California San Diego Human Subjects Protocol) and cultured as previously reported [21,22]. GBM4 and 8 cells were a kind gift from C. David James (University of California San Francisco). Briefly, the dissociated tissue was washed, filtered through a $30 \mu \mathrm{m}$ mesh and plated onto ultra-low adherence flasks at a concentration of 500,000 to $1,500,000$ viable cells $/ \mathrm{ml}$. The stem cell isolation medium included human recombinant EGF (20 $\mathrm{ng} / \mathrm{ml})$, human bFGF $(10 \mathrm{ng} / \mathrm{ml})$ and heparin $(2 \mu \mathrm{g} / \mathrm{ml})$. Sphere cultures were passaged by dissociation using Acutase (Sigma), washed, resuspended in neural stem cell culture medium (\#05750, Stemcell Technologies), and plated on ultra low-adherence 96 well plates at 2000 cells per well for all subsequent drug testing. We characterized all patient-derived glioblastoma lines using histopathologic and integrated genomic analyses. The glioblastoma lines were profiled using the Affymetrix Gene Chip Human Gene 1.0 ST Array.

\section{Drug screening}

Drug screens were performed on patient-derived GBM cell lines plated at 2000 cell per well in 96-well microtiter plates, incubated overnight. After 72 hours of incubation with drugs, cell viability was quantified by the Alamar Blue assay. Briefly, after incubation, Alamar Blue (\#BUF012B, AbDSerotec) was added directly to the culture medium, and the fluorescence measured at 560/90 to determine the number of viable cells (Infinite M200, Tecan Group Ltd.).

\section{Results}

Our study involved a retrospective component where we predicted gene mutations - drug sensitivity associations defined in a recent hypothesis-independent study [23] In addition, we predicted sensitivity of our profiled patient-derived GBM cell lines to targeted agents and compared these in silico predictions to in vitro experimental data.

\section{Retrospective validation of in Silico tumor model}

In the first part of the study, we evaluated the ability of the in silico tumor model to predict drug responses that were reported in the study by Garnett and colleagues [23]. A comparison of our predictions with the associations reported in the Garnett study indicated the predictive capability of our in silico tumor model.

Our modeling library has definitions for 45 of the 639 cell lines used in this study (Additional file 1: Table S2) and supports 70 of the 130 drugs studied (Additional file 1: Table S3). Further, we can represent 51 of the 84 genes screened for mutations (Additional file 1: Table S4). Of the 448 significant gene mutation-drug response associations reported, our in silico model was able to accurately predict 22 of the 25 testable associations from the Garnett study ( $>85 \%$ agreement; Additional file 1: Table S5). The gene mutation-drug response correlations from the Garnett study that are currently not supported by the system are listed in Additional file 1: Table S6. From the 25 gene mutation-drug response associations tested from the Garnett study (Additional file 1: Table S5), a few examples of the correlations are explained below. Figure 1A depicts a representative schematic of this retrospective analysis using the simulation (in silico tumor model).

\section{BRAF Mutations and Drug Sensitivity}

The Garnett study showed that cells with BRAF mutation were sensitive to the MEK1/2 inhibitor AZD2644 [23]. To examine this association, we modeled cancer cell variants with wild-type BRAF in silico. Modeling data showed that cells with wild-type BRAF were resistant to AZD6244, when compared to the parent tumor cells with mutant BRAF. Thus, BRAF mutation conferred sensitivity to the MEK1/2 inhibitor in silico; this prediction validates the finding reported in the Garnett study (Figure 1A). 40-60\% melanoma patients carry BRAF mutations that activate MAPK signaling $[24,25]$ and this association could have therapeutic implications for the treatment of patients with BRAF mutant melanoma. 


\section{Effect of different mutations on sensitivity to tyrosine Kinase inhibitors}

The Garnett study showed that cells with BRAF mutation were sensitive to the MEK1/2 inhibitor AZD2644 [23]. To examine this association, we created cancer cell variants with wild-type BRAF in the in silico model. Simulation data showed that cells with wild-type BRAF were resistant to AZD6244, when compared to cells with mutant BRAF. Thus, BRAF mutation conferred sensitivity to the MEK1/2 inhibitor; this validates the finding reported in the Garnett study (Figure 2A). 40-60\% melanoma patients carry BRAF mutations that activate MAPK signaling $[24,25]$. This association tested in Figure 2A may have therapeutic implications for the treatment of patients with BRAF mutant melanoma.

ERBB2 (HER2) amplification is a biomarker for sensitivity to EGFR-family inhibitors [26]. In the in silico model, we tested for sensitivity to EGFR2 family inhibitors, lapatinib and BIBW2992. Specifically, we examined sensitivity of cancer cells in the presence of mutations and/or over-expression of BRAF, CDH1, ERBB2, CCND1 and MET. These predictions from simulations were compared with results obtained in the Garnett study and the predictive capability of our model was determined.

In silico predictions indicate that BRAF mutation decreases sensitivity of cells to lapatinib (Figure 2B), whereas $\mathrm{CDH} 1$ mutant lines demonstrated higher sensitivity to lapatinib when compared to variants with wild-type CDH1 (Figure 2C). Further, cMET over-expression showed increased sensitivity to lapatinib, as indicated by decrease in viability in cells with cMET over-expression (Figure 2D). Additionally, ERBB2 and CCND1 over-expression correlated positively with lapatinib sensitivity (Additional file 1: Table S5). In all these simulation experiments
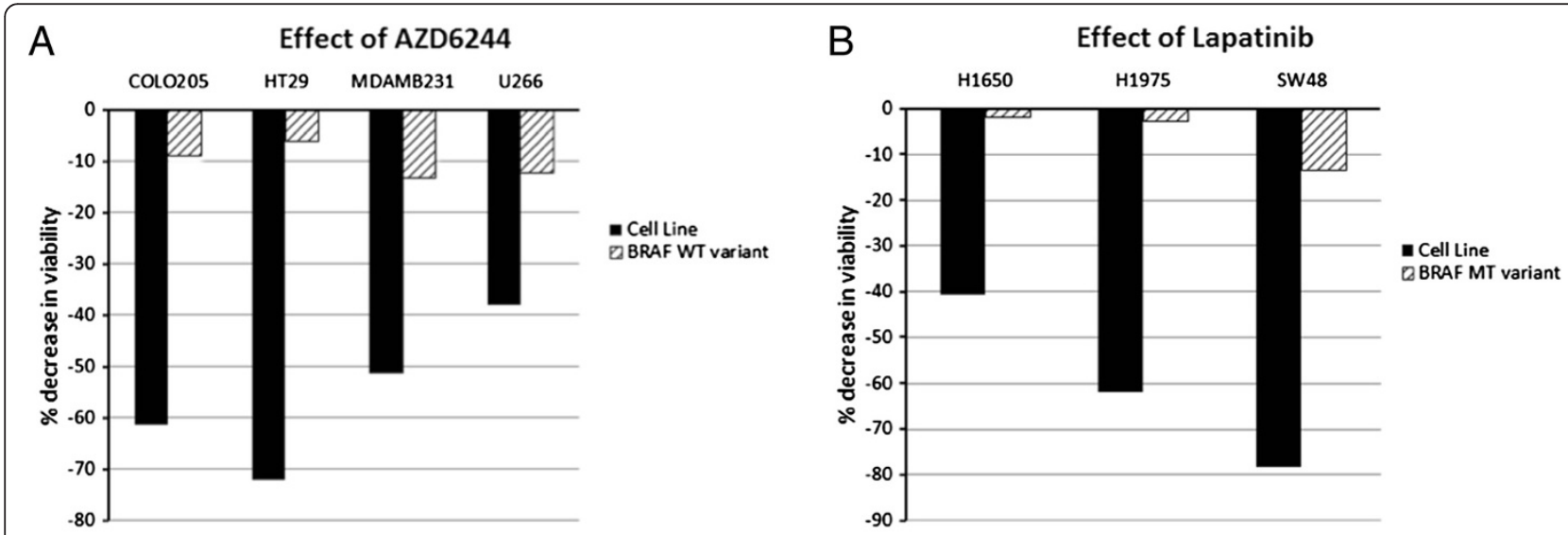

C $\quad$ Effect of Lapatinib

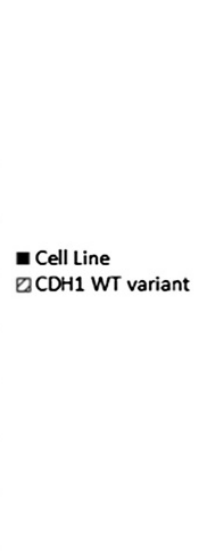

D

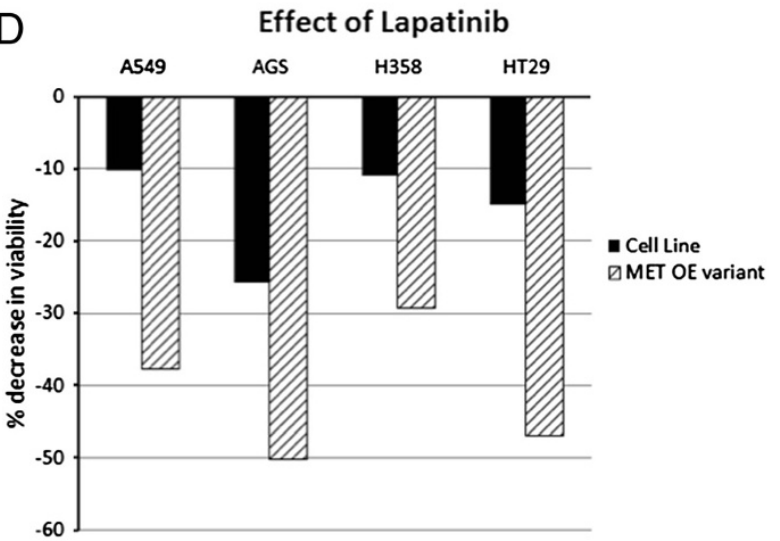

Figure 2 Retrospective analysis tests in silico predictions of gene mutations and sensitivity to EGFR family inhibitors. Associations reported in the Garnett study were tested in a blinded manner using our in silico model and predictions obtained were compared to results reported in the Garnett study. A, We created wild-type BRAF variants of four cancer cell lines - COLO205, HT29, MDAMB231 and U266 in silico and compared the effect of MEK1/2 inhibitor AZD2644 on these cell lines and on corresponding parent lines expressing mutant BRAF. Our data demonstrated that BRAF mutation increases sensitivity to AZD6244. B. We simulated three cell lines - H1650, H1975 and SW48 with wild-type or mutant BRAF and tested for sensitivity to the EGFR2 family kinase inhibitor, lapatinib. BRAF mutation decreases sensitivity of cells to lapatinib. C, Similarly, when four cell lines (AGS, H1437, MKN1 and MKN45) were tested for sensitivity to lapatinib, we observed that CDH1 mutation increases sensitivity to lapatinib. D, We generated cell lines with wild-type or MET over-expression and tested the effect of lapatinib (A549, AGS, H358 and HT29 cell lines). MET over-expression increases sensitivity to lapatinib. 
testing sensitivity to lapatinib, our in silico predictions corroborated with associations reported in the Garnett study.

\section{CDKN2A mutation and drug sensitivity}

The Garnett study reported associations between tumor suppressor gene mutations and several anti-cancer drugs. We tested these associations in our in silico tumor model. In the in silico analysis, cells harboring wild-type CDKN2A were resistant to erlotinib whereas CDKN2A mutation was associated with erlotinib sensitivity (Figure 3A). Similarly, cell lines with mutant CDKN2A showed increased sensitivity to dasatinib (Figure 3B), bortezomib (Figure 3C), and to the CDK4/6 inhibitor PD0332991 (Figure 3D). These predictions/analyses from our simulation corroborated accurately with data from the Garnett study.

Other gene mutation-drug response associations examined in our simulation models are illustrated in Additional file 1: Table S5. In addition, Additional file 1: Table S6 lists correlations between gene mutations and drug responses reported in the Garnett study, which are currently not supported by our modeling technology. In spite of these limitations, we obtained $\sim 85 \%$ agreement of our simulation data with findings reported by Garnett [23].

\section{Prospective evaluation of tumor model - patient-derived GBM cell lines}

Identifying drug sensitivities in tumors/cancers with different mutations is important for designing individualized therapies for cancer. To this end, we created in silico avatars of 8 patient-derived GBM cell lines using genomic data (Methods and Additional file 1: Table S1) and predicted their sensitivity to various targeted therapeutic agents. We then tested these in silico predictions prospectively by comparing then with experimental data obtained by in vitro testing on the same patient-derived GBM cell lines (Figure 1B).

The patient-derived GBM cell lines were obtained from patient tumors resected surgically and cultured in vitro

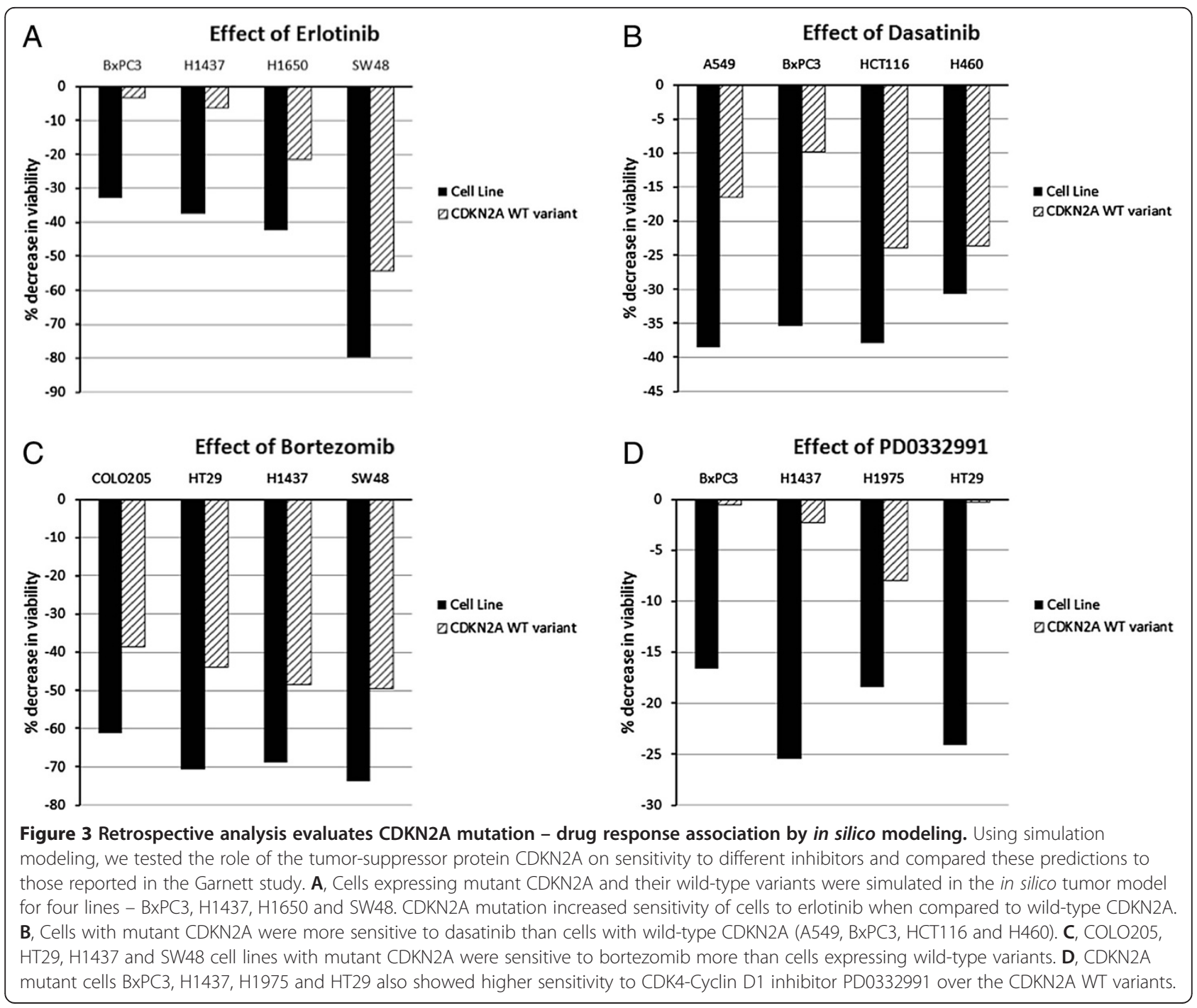


(details in Methods). We have profiled these lines using Affymetrix Gene Chip Human Gene 1.0 ST Array. Using whole-exome sequencing, we recently tested the validity of these cells (maintained in cultures) for development and testing of personalized targeted therapies, based on their accurate representation of the original tumor profiles [27]. We have designated the different patient-derived
GBM cell lines as: GBM4, GBM8, SK102, SK262, SK429, SK748, SK987 and SK1035.

After generating in silico profiles of these cells, we optimized these simulation avatars in terms of strength of functional effect of the mutation on key pathways such as EGFR, RAS and Src/PI3K. The rationale for this optimization is that expression data on these cells does not provide an

\section{A}

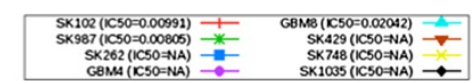

vabiliy Ourve whe ncreasing Dose

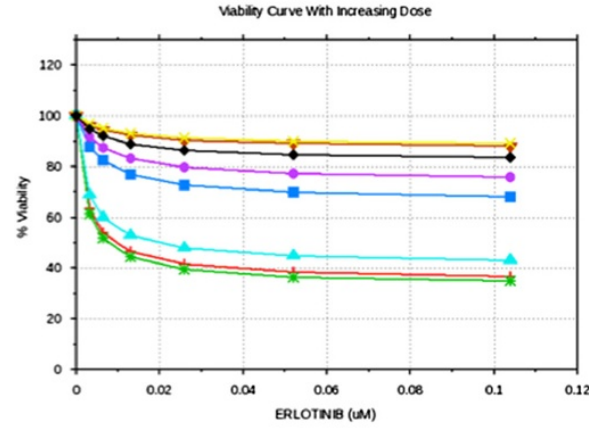

C

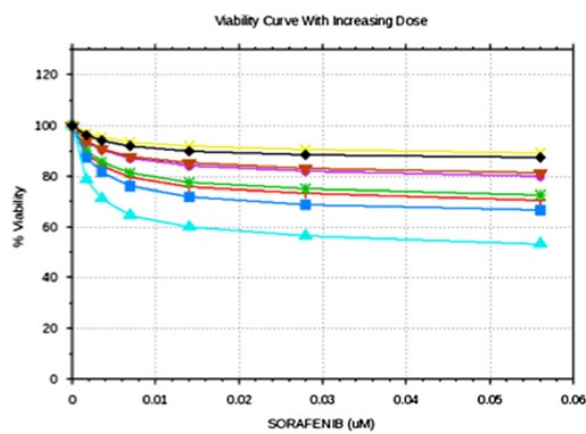

E
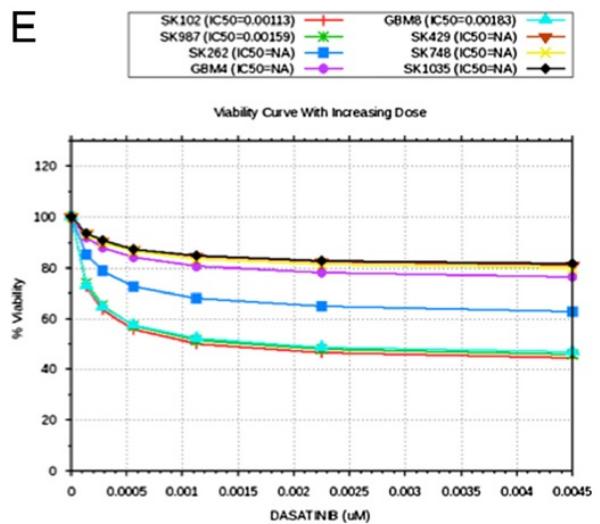

B

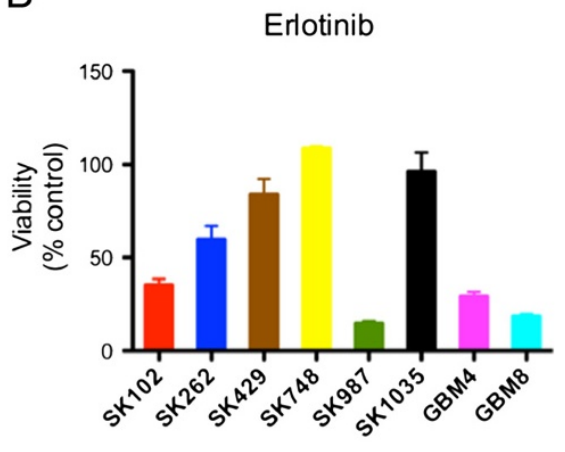

Sorafenib

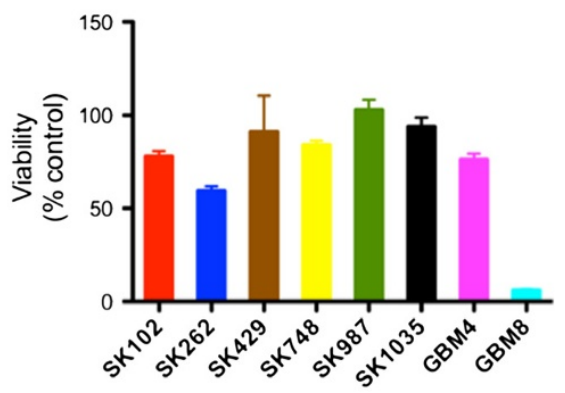

$\mathrm{F}$

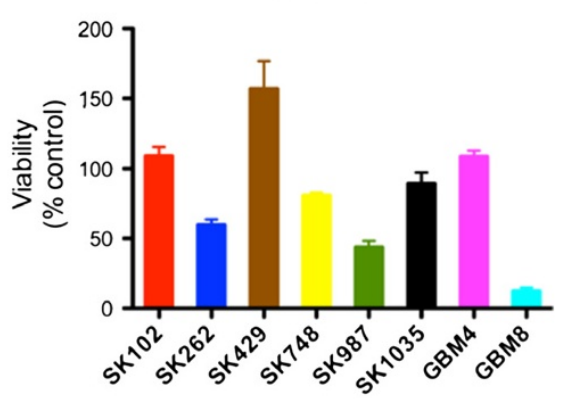

Figure 4 In silico modeling analysis and experimental in vitro data for drug responsiveness to 3 alignment drugs. A, Predictive dose response data for erlotinib with percent change in viability. Cells showing decrease in viability of $20 \%$ or greater are considered sensitive to the drug. B, In vitro experimental results for effect of $1 \mu \mathrm{M}$ erlotinib on viability in patient-derived GBM cell lines; viability was determined at $72 \mathrm{~h}$ using Alamar Blue assay. C, D, Predictive and experimental data for sorafenib. E, F, Predictive and experimental data for dasatinib. All drugs were tested in vitro at $1 \mu \mathrm{M}$. Dose-response curves for in silico data demonstrate the effects of increasing concentrations of the drugs - erlotinib, sorafenib and sunitinib on the viability of profiled patient-derived GBM cell lines in the simulation model. 

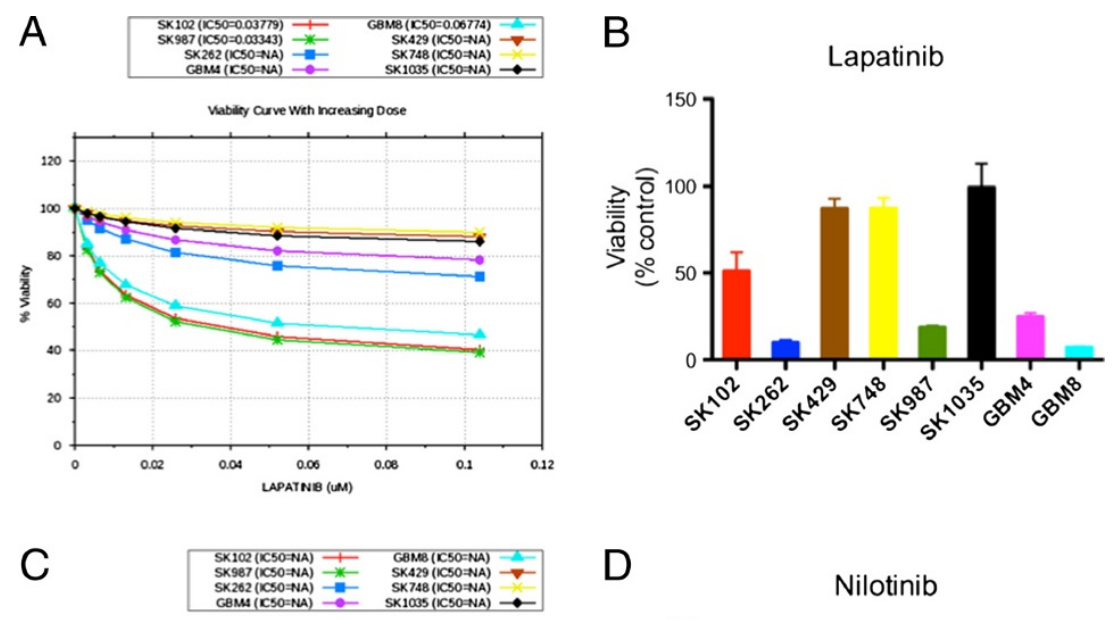

D Nilotinib
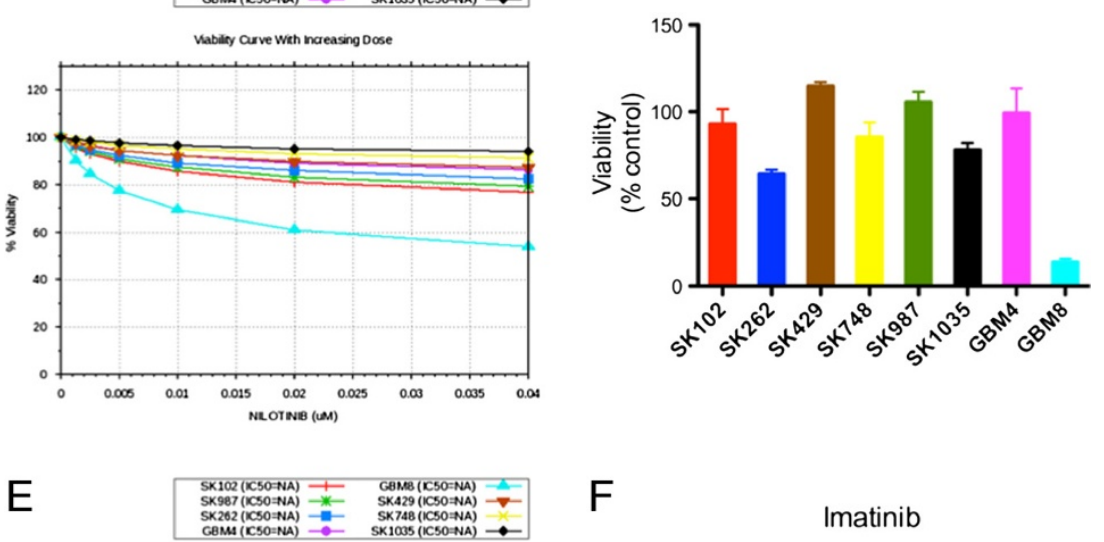

F
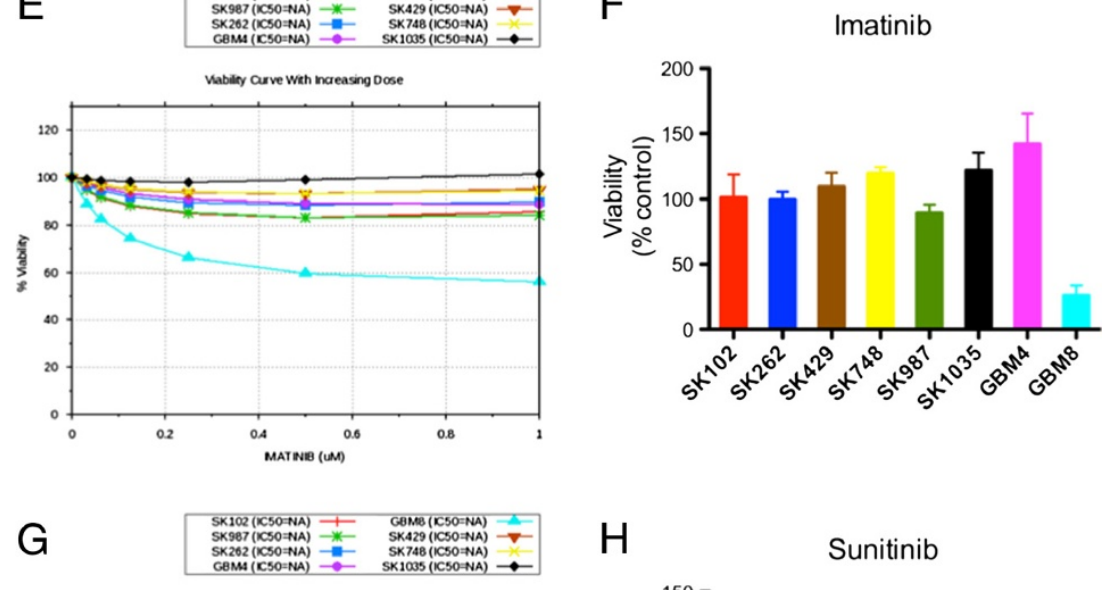

$\mathrm{H}$
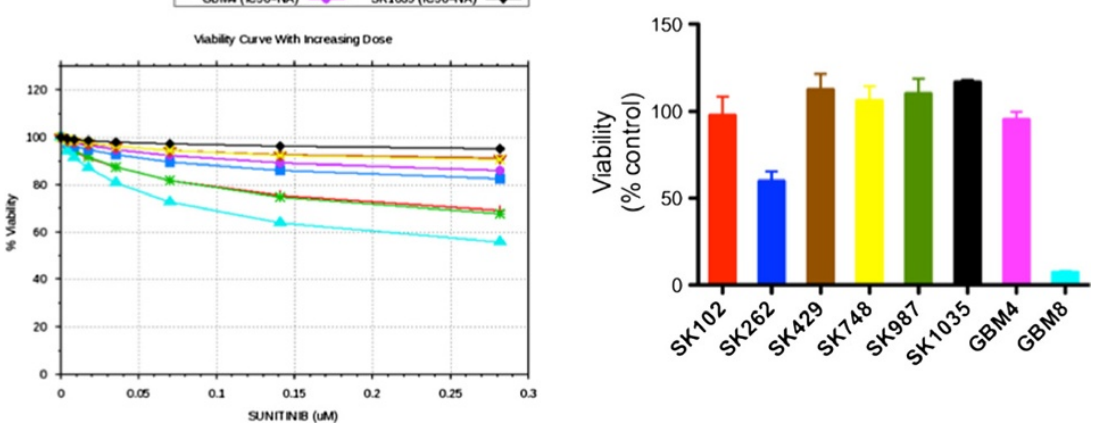

Figure $\mathbf{5}$ (See legend on next page.) 
accurate measure of the dominance of different intracellular pathways. In order to interrogate this information on the pathways that play a dominant role in each tumor line (such as EGFR, RAS, PI3K, etc.), we used 3 anti-cancer agents (erlotinib, sorafenib and dasatinib) targeting these pathways. This will achieve "alignment" and train the simulation avatars for further analyses (details in Additional file 1). The alignment for these 3 drugs could be best achieved in the following cell lines: GBM8, SK262, SK429, SK748, and SK1035. In cell lines GBM4 and SK987, there was a mismatch for sorafenib where the predictive trends were reversed. GBM4 was sensitive to sorafenib experimentally but our in silico predictions showed it to be resistant; SK987 was resistant experimentally but sensitive in predictive results. Similarly, the experimental trend for SK102 resistance to dasatinib could not be met predictively. Correlation of predictive trends with alignment drugs is shown in Figure 4 A-F.

Predictions obtained by simulation modeling are presented as dose-response plots for viability; decrease in viability of $>20 \%$ was considered as sensitive. Experimentally, viability was determined by Alamar blue assay, in response to $1 \mu \mathrm{M}$ concentration of respective inhibitors at $72 \mathrm{~h}$. These data represent viability as mean values from triplicate samples.

We tested ten anti-cancer drugs in silico on the simulation avatars of the 8 patient-derived GBM cell lines in a blinded prospective study. These simulations generated predictions that we compared with in vitro experimental data (Additional file 1: Table S7A-D). Of the 80 in silico predictions, $61(76.25 \%)$ predictions showed agreement with in vitro experimental results. Analysis of drug sensitivity correlation for all $8 \mathrm{GBM}$ patient-derived cell lines, for all the 13 drugs is summarized in Additional file 1: Table S7. Figures 5A-H and 6A-H show a drugwise comparison of in silico predictions (dose-response curves) and in vitro experimental results generated with testing $1 \mu \mathrm{M}$ concentration of each drug on these cell lines.

\section{Effect of tyrosine kinase inhibitors on patient-derived GBM cells}

For the EGFR family inhibitor lapatinib, simulation studies predicted SK429, SK748 and SK1035 to be resistant, which were confirmed by in vitro data. Similarly, modeling predicted GBM8, SK102, SK262 and SK987 to be sensitive and these predictions were in agreement with experimental data (Figure 5A and B). However, modeling predicted GBM4 to be resistant to lapatinib while in vitro data showed GBM4 to be highly sensitive to lapatinib (Figure 5B). For the tyrosine kinase inhibitor nilotinib, the model predicted GBM8 to be sensitive while all the other profiles to be resistant (Figure $5 \mathrm{C}$ ). In vitro studies demonstrated that GBM8 was indeed sensitive to nilotinib as predicted, but there was a mismatch with the experimental results for two lines - SK262 and SK1035. Experimentally, SK262 was found to be sensitive, whereas SK1035 was on the borderline of sensitivity and resistance (Figure 5D). For imatinib, simulation predicted that all GBM lines except GBM8 were resistant (Figure 5E). The experimental results corroborated with this in silico prediction (Figure 5F). Sunitinib was the other multi-tyrosine kinase inhibitor tested. Our simulation predicted GBM8, SK102 and SK987 to be sensitive to sunitinib; however, only GBM8 was found to be sensitive in vitro. SK262 was predicted to be resistant to sunitinib but in vitro data found it to be moderately sensitive. On the other hand, GBM4, SK429, SK748 and SK1035 were found to be resistant in both simulation and experimental data (Figure 5G-H).

\section{Effect of other drugs on patient-derived GBM cells}

Besides the tyrosine kinase inhibitors, correlation between in silico predictions and experimental results for the 8 patient-derived GBM cell lines was also tested for drugs such as pitavastatin (HMG CoA reductase inhibitor), everolimus (mTOR inhibitor), celecoxib (COX2 inhibitor) and bortezomib (proteasome inhibitor) (Figure 6 A-H). For bortezomib, all profiles were predicted to be sensitive and these predictions matched with in vitro experimental results (Figure 6A and B). For everolimus, in vitro results were in agreement with simulation predictions for all lines except SK429 (Figure 6C and D). Our in silico model predicted GBM4, SK262, SK429, SK748 and SK1035 to be resistant to celecoxib; these predictions matched with in vitro results. However, GBM8, SK102 and SK987 were predicted to show moderate sensitivity to celecoxib, but were found to be resistant in vitro (Figure $6 \mathrm{E}$ and $\mathrm{F}$ ). For pitavastatin, the simulation predicted 5 patient-derived GBM cell lines to be sensitive (GBM8, GBM4, SK102, SK262 and SK987), of which SK987 was found to be resistant in vitro. On the other 
A
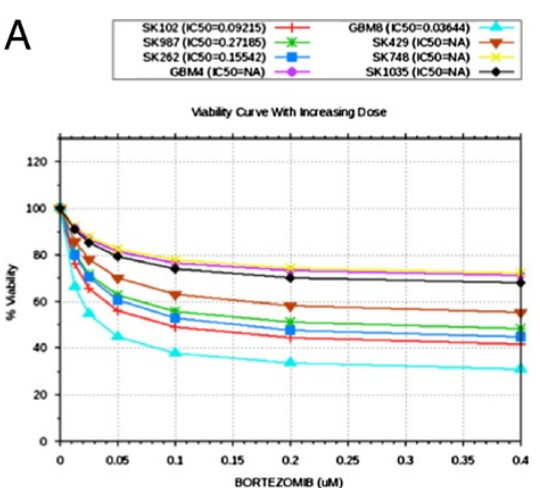

C

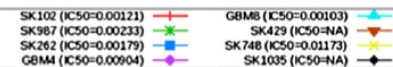

Mabiley arve Weh inceasing Dose

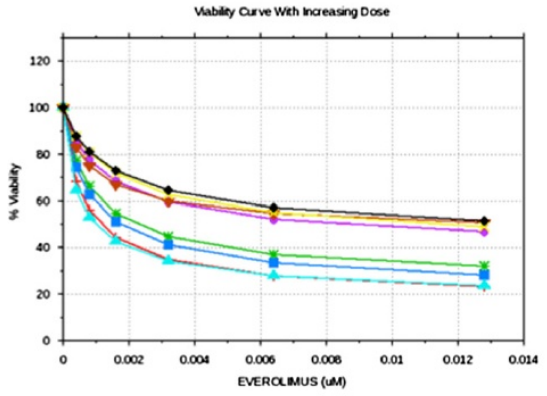

$E$

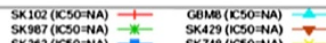

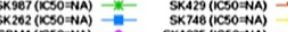
GBMA (CSO=NA) - SK1005 (ICSONAA) -
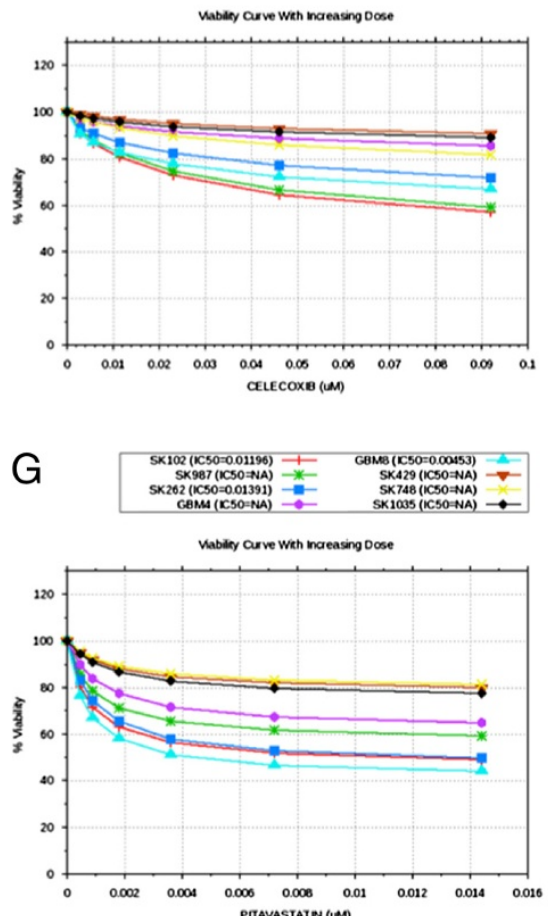

Figure 6 (See legend on next page.)
B Bortezomib

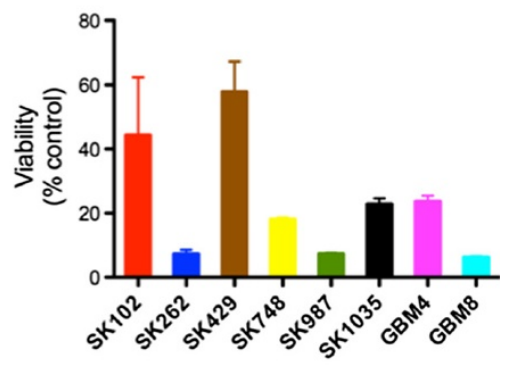

D

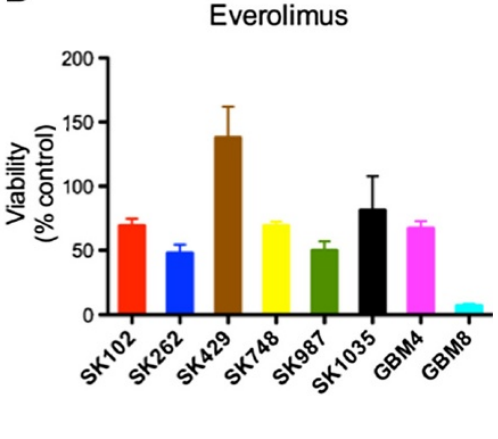

$\mathrm{F}$

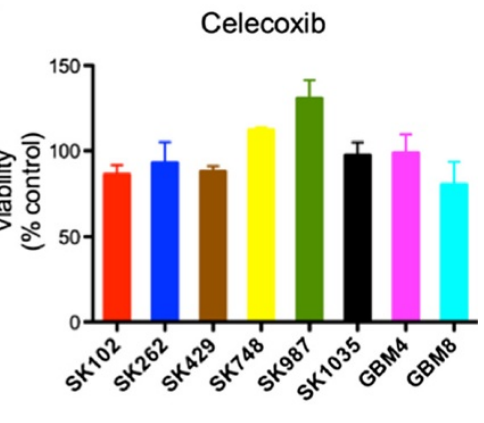

$\mathrm{H}$

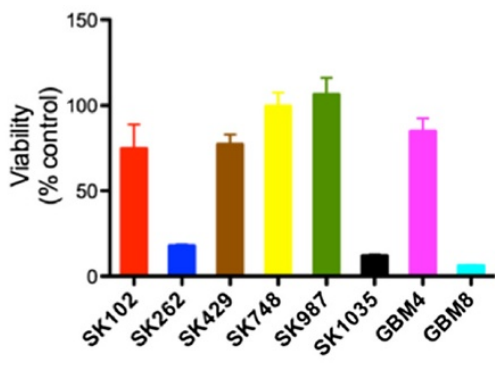


hand, of the cell lines predicted to be resistant (SK429, SK748 and SK1035), SK1035 was sensitive in vitro and did not match with the prediction (Figure 6G and $\mathrm{H}$ ).

These data demonstrate a $76.25 \%$ agreement between in silico predictions of drug response and in vitro experimental data in patient-derived GBM cell lines.

\section{Discussion}

Developing an in silico model that takes into account the complex genotypes/phenotypes of cancer to accurately predict drug response will help personalize therapy with more efficiency. In this study, we developed and validated a virtual tumor model by retrospectively testing it against a dataset from a recent screening study [23]; we obtained a corroboration of $\sim 85 \%$ between our predictions and the results from this study. Following this retrospective validation, we generated in silico predictions to prospectively test the sensitivity of patientderived GBM cell lines to targeted agents. These analyses also demonstrated a high degree of agreement $(>75 \%)$ between in vitro experimental findings and in silico predictions. These studies validate our in silico tumor model and the simulation-based approach and provide critical proof-of-concept of a priori prediction of responses to targeted therapies. Thus, this model provides an effective platform for testing and developing personalized therapeutic regimens for cancer patients.

The genomic inputs that we used to create simulation avatars for patient-derived GBM cell lines were copy number variation data. A more comprehensive and accurate profile would require additional data (gene mutations, methylation status etc. along with copy number variation); this would help us develop a more representative avatar and would likely improve the accuracy of our drug response predictions and provide higher correlation with experimental data.

Genotypes of cancer cell lines have traditionally been used to correlate with drug sensitivity $[28,29]$. A similar recent study makes efficient use of gene expression profiles to categorize colorectal cancers into different molecular and clinically actionable subtypes [30]. Moreover, it is clear that using molecular tumor profiles to stratify patients for therapy affects response and progressionfree survival [31]. However, increasing amounts of data from genomic, proteomic, transcriptomic and metabolomic profiling will likely require integration of these varied datasets and development of predictive systems modeling, which may hold the key to effective cancer therapy.

Rapid screening of patient samples in real time with models such as the one we have developed can drive critical therapeutic decision-making. Although our current model makes only cell-intrinsic predictions, we have been able to achieve a high rate of agreement between in silico predictions and in vitro findings. Future versions of this model are being refined to incorporate tumor microenvironment including aspects of angiogenesis, hypoxia, and tumor-associated inflammation. We believe that incorporating these features into our model would more accurately represent the tumor in a patient. Importantly, this will further help improve our predictions for designing therapeutic regimens for GBM patients. This model can also be adapted to identify potential mechanisms of resistance $a$ priori and to design rational drug combinations that prevent emergence of resistance and development of escape pathways.

Our in silico model aligns with NCI guidelines that emphasize evaluation of similar predictor models to determine their accuracy $[12,15,32,33]$. We intend to test this model in clinical trials and utilize it as a tool to expedite clinical decision-making and determine drugs/ combinations most likely to benefit a patient. Additionally, models such as these will play important roles in testing new biological hypotheses. This is critical to the discovery of molecular drivers and critical networks in cancer pathophysiology and the development of better diagnostics and effective therapeutics.

\section{Additional file}

Additional file 1: Supplementary Information.

\section{Competing interests}

The following authors are employed by Cellworks, Inc.: Zeba Sultana, Taher Abbasi, Shweta Kapoor, Ansu Kumar, Shahabuddin Usmani, Ashish Agrawal, and Shireen Vali. The other authors report no competing financial interests.

\section{Authors' contributions}

SCP - designed study, performed research, analyzed data, wrote manuscript; ZS - executed simulation studies, analyzed data, wrote manuscript; SP - designed study, performed research, analyzed data, wrote manuscript; PJ - performed research, analyzed data; RM - performed research, analyzed data; YC - performed research, analyzed data; ISB - performed research; NN - performed research; MM - performed research, analyzed data; TA - analyzed data, developed analytics, wrote manuscript; SK - developed predictive simulation-based tumor cell technology; AK - developed predictive simulation-based tumor cell technology; SU - executed simulation studies, developed predictive simulation-based tumor cell technology; AA - developed predictive simulation-based tumor cell technology; SV - analyzed data, 
developed predictive simulation-based tumor cell technology, developed analytics, wrote manuscript; SK (Santosh Kesari) - designed study, planned and directed research, analyzed data, wrote manuscript. All authors read and approved the final manuscript.

\section{Acknowledgments}

This work was supported in part by grants from National Brain Tumor Society (NBTS) David Cook Chair of Research, Barbara and Joseph Ajello trust fund, Tuttleman Family Foundation, MCJ Amelior Foundation, and Boston Fire Department/Kenney Foundation to S. Kesari.

\section{Author details}

${ }^{1}$ Translational Neuro-Oncology Laboratories, Moores Cancer Center, UC San Diego, La Jolla, CA 92093, USA. ${ }^{2}$ Cellworks Research India Ltd, Bangalore 560 066, India. ${ }^{3}$ Cellworks Group Inc, Saratoga, CA 95070, USA. ${ }^{4}$ Department of Neurosciences, UC San Diego, La Jolla, CA 92093, USA.

Received: 14 January 2014 Accepted: 23 April 2014

Published: 21 May 2014

\section{References}

1. Wen PY, Kesari S: Malignant gliomas in adults. N Engl J Med 2008, 359:492-507.

2. Mischel PS, Shai R, Shi T, Horvath S, Lu KV, Choe G, Seligson D, Kremen TJ, Palotie A, Liau LM, Cloughesy TF, Nelson SF: Identification of molecular subtypes of glioblastoma by gene expression profiling. Oncogene 2003, 22:2361-2373

3. TCGA: Comprehensive genomic characterization defines human glioblastoma genes and core pathways. Nature 2008, 455:1061-1068

4. Verhaak RG, Hoadley KA, Purdom E, Wang V, Qi Y, Wilkerson MD, Miller CR, Ding L, Golub T, Mesirov JP, Alexe G, Lawrence M, O'Kelly M, Tamayo P, Weir BA, Gabriel S, Winckler W, Gupta S, Jakkula L, Feiler HS, Hodgson JG, James CD, Sarkaria JN, Brennan C, Kahn A, Spellman PT, Wilson RK, Speed TP, Gray JW, Meyerson M, et al: Integrated genomic analysis identifies clinically relevant subtypes of glioblastoma characterized by abnormalities in PDGFRA, IDH1, EGFR, and NF1. Cancer Cell 2010, 17:98-110.

5. Deisboeck TS: Personalizing medicine: a systems biology perspective. $\mathrm{Mol}$ Syst Biol 2009, 5:249.

6. Deisboeck TS, Zhang L, Yoon J, Costa J: In silico cancer modeling: is it ready for prime time? Nat Clin Pract Oncol 2009, 6:34-42.

7. Woodward DE, Cook J, Tracqui P, Cruywagen GC, Murray JD, Alvord EC Jr: A mathematical model of glioma growth: the effect of extent of surgical resection. Cell Prolif 1996, 29:269-288.

8. Tracqui P, Cruywagen GC, Woodward DE, Bartoo GT, Murray JD, Alvord EC $\mathrm{Jr}$ : A mathematical model of glioma growth: the effect of chemotherapy on spatio-temporal growth. Cell Prolif 1995, 28:17-31.

9. Zhang L, Wang Z, Sagotsky JA, Deisboeck TS: Multiscale agent-based cancer modeling. J Math Biol 2009, 58:545-559.

10. Deisboeck TS, Wang Z, Macklin P, Cristini V: Multiscale cancer modeling. Annu Rev Biomed Eng 2011, 13:127-155.

11. Almine JF, Wise SG, Hiob M, Singh NK, Tiwari KK, Vali S, Abbasi T, Weiss AS: Elastin sequences trigger transient proinflammatory responses by human dermal fibroblasts. FASEB J 2013, 27:3455-3465.

12. Barve A, Gupta A, Solapure SM, Kumar A, Ramachandran V, Seshadri K, Vali S, Datta S: A kinetic platform for in silico modeling of the metabolic dynamics in Escherichia coli. Adv Appl Bioinform Chem 2010, 3:97-110.

13. Cirstea D, Hideshima T, Rodig S, Santo L, Pozzi S, Vallet S, Ikeda H, Perrone G Gorgun G, Patel K, Desai N, Sportelli P, Kapoor S, Vali S, Mukherjee S, Munshi NC, Anderson $\mathrm{KC}$, Raje N: Dual inhibition of akt/mammalian target of rapamycin pathway by nanoparticle albumin-bound-rapamycin and perifosine induces antitumor activity in multiple myeloma. Mol Cancer Ther 2010, 9:963-975

14. Equils O, Nambiar P, Hobel CJ, Smith R, Simmons CF, Vali S: A computer simulation of progesterone and Cox2 inhibitor treatment for preterm labor. PLoS One 2010, 5:e8502.

15. Harvey LE, Kohlgraf KG, Mehalick LA, Raina M, Recker EN, Radhakrishnan S, Prasad SA, Vidva R, Progulske-Fox A, Cavanaugh JE, Vali S, Brogden KA: Defensin DEFB103 bidirectionally regulates chemokine and cytokine responses to a pro-inflammatory stimulus. Sci Rep 2013, 3:1232.

16. Kannaiyan R, Hay HS, Rajendran P, Li F, Shanmugam MK, Vali S, Abbasi T, Kapoor S, Sharma A, Kumar AP, Chng WJ, Sethi G: Celastrol inhibits proliferation and induces chemosensitization through down-regulation of NF-kappaB and STAT3 regulated gene products in multiple myeloma cells. Br J Pharmacol 2011, 164:1506-1521.

17. Kaushik P, Gorin F, Vali S: Dynamics of tyrosine hydroxylase mediated regulation of dopamine synthesis. J Comput Neurosci 2007, 22:147-160

18. Tandon R, Kapoor S, Vali S, Senthil V, Nithya D, Venkataramanan R, Sharma A, Talwadkar A, Ray A, Bhatnagar PK, Dastidar SG: Dual epidermal growth factor receptor (EGFR)/insulin-like growth factor-1 receptor (IGF-1R) inhibitor: a novel approach for overcoming resistance in anticancer treatment. Eur J Pharmacol 2011, 667:56-65.

19. Vali S, Mythri RB, Jagatha B, Padiadpu J, Ramanujan KS, Andersen JK, Gorin F, Bharath MM: Integrating glutathione metabolism and mitochondrial dysfunction with implications for Parkinson's disease: a dynamic model. Neuroscience 2007, 149:917-930

20. Vali S, Pallavi R, Kapoor S, Tatu U: Virtual prototyping study shows increased ATPase activity of $\mathrm{Hsp} 90$ to be the key determinant of cancer phenotype. Syst Synth Biol 2010, 4:25-33.

21. Galli R, Binda E, Orfanelli U, Cipelletti B, Gritti A, De Vitis S, Fiocco R, Foroni C, Dimeco F, Vescovi A: Isolation and characterization of tumorigenic, stem-like neural precursors from human glioblastoma. Cancer Res 2004, 64:7011-7021

22. Lee J, Kotliarova S, Kotliarov Y, Li A, Su Q, Donin NM, Pastorino S, Purow BW, Christopher N, Zhang W, Park JK, Fine HA: Tumor stem cells derived from glioblastomas cultured in bFGF and EGF more closely mirror the phenotype and genotype of primary tumors than do serum-cultured cell lines. Cancer Cell 2006, 9:391-403.

23. Garnett MJ, Edelman EJ, Heidorn SJ, Greenman CD, Dastur A, Lau KW, Greninger P, Thompson IR, Luo X, Soares J, Liu Q, lorio F, Surdez D, Chen L, Milano RJ, Bignell GR, Tam AT, Davies H, Stevenson JA, Barthorpe S, Lutz SR, Kogera F, Lawrence K, McLaren-Douglas A, Mitropoulos X, Mironenko T, Thi H, Richardson L, Zhou W, Jewitt F, et al: Systematic identification of genomic markers of drug sensitivity in cancer cells. Nature 2012, 483:570-575.

24. Curtin JA, Fridlyand J, Kageshita T, Patel HN, Busam K, Kutzner H, Cho KH, Aiba S, Brocker EB, LeBoit PE, Pinkel D, Bastian BC: Distinct sets of genetic alterations in melanoma. N Engl J Med 2005, 353:2135-2147.

25. Davies H, Bignell GR, Cox C, Stephens P, Edkins S, Clegg S, Teague J, Woffendin H, Garnett MJ, Bottomley W, Davis N, Dicks E, Ewing R, Floyd Y, Gray K, Hall S, Hawes R, Hughes J, Kosmidou V, Menzies A, Mould C, Parker A, Stevens C, Watt S, Hooper S, Wilson R, Jayatilake H, Gusterson BA, Cooper C, Shipley J, et al: Mutations of the BRAF gene in human cancer. Nature 2002, 417:949-954.

26. Konecny GE, Pegram MD, Venkatesan N, Finn R, Yang G, Rahmeh M, Untch M Rusnak DW, Spehar G, Mullin RJ, Keith BR, Gilmer TM, Berger M, Podratz KC, Slamon DJ: Activity of the dual kinase inhibitor lapatinib (GW572016) against HER-2-overexpressing and trastuzumab-treated breast cancer cells. Cancer Res 2006, 66:1630-1639.

27. Yost SE, Pastorino S, Rozenzhak S, Smith EN, Chao YS, Jiang P, Kesari S, Frazer KA, Harismendy O: High-resolution mutational profiling suggests the genetic validity of glioblastoma patient-derived pre-clinical models. PLoS One 2013, 8:e56185.

28. Shoemaker RH, Monks A, Alley MC, Scudiero DA, Fine DL, McLemore TL, Abbott BJ, Paull KD, Mayo JG, Boyd MR: Development of human tumor cell line panels for use in disease-oriented drug screening. Prog Clin Biol Res 1988, 276:265-286.

29. Weinstein JN, Myers TG, O'Connor PM, Friend SH, Fornace AJ Jr, Kohn KW, Fojo T, Bates SE, Rubinstein LV, Anderson NL, Buolamwini JK, van Osdol WW, Monks AP, Scudiero DA, Sausville EA, Zaharevitz DW, Bunow B, Viswanadhan VN, Johnson GS, Wittes RE, Paull KD: An information-intensive approach to the molecular pharmacology of cancer. Science 1997, 275:343-349.

30. Sadanandam A, Lyssiotis CA, Homicsko K, Collisson EA, Gibb WJ, Wullschleger S, Ostos LC, Lannon WA, Grotzinger C, Del Rio M, Lhermitte B, Olshen AB, Wiedenmann B, Cantley LC, Gray JW, Hanahan D: A colorectal cancer classification system that associates cellular phenotype and responses to therapy. Nat Med 2013, 19:619-625.

31. Von Hoff DD, Stephenson JJ Jr, Rosen P, Loesch DM, Borad MJ, Anthony S, Jameson G, Brown S, Cantafio N, Richards DA, Fitch TR, Wasserman E, Fernandez C, Green S, Sutherland W, Bittner M, Alarcon A, Mallery D, Penny R: Pilot study using molecular profiling of patients' tumors to find potential targets and select treatments for their refractory cancers. J Clin Oncol 2010, 28:4877-4883. 
32. Rajendran P, Ong TH, Chen L, Li F, Shanmugam MK, Vali S, Abbasi T, Kapoor S, Sharma A, Kumar AP, Hui KM, Sethi G: Suppression of signal transducer and activator of transcription 3 activation by butein inhibits growth of human hepatocellular carcinoma in vivo. Clin Cancer Res 2011, 17:1425-1439.

33. Sultana Z, Paleologou KE, Al-Mansoori KM, Ardah MT, Singh N, Usmani S, Jiao H, Martin FL, Bharath MM, Vali S, El-Agnaf OM: Dynamic modeling of alpha-synuclein aggregation in dopaminergic neuronal system indicates points of neuroprotective intervention: experimental validation with implications for Parkinson's therapy. Neuroscience 2011, 199:303-317.

doi:10.1186/1479-5876-12-128

Cite this article as: Pingle et al.: In silico modeling predicts drug

sensitivity of patient-derived cancer cells. Journal of Translational Medicine 2014 12:128.

\section{Submit your next manuscript to BioMed Central and take full advantage of:}

- Convenient online submission

- Thorough peer review

- No space constraints or color figure charges

- Immediate publication on acceptance

- Inclusion in PubMed, CAS, Scopus and Google Scholar

- Research which is freely available for redistribution 